\title{
Intramedullary Spinal Cysticercosis: A Case Report and Review of Literature
}

\author{
Moinay Kim", Seung Chul Rhim ${ }^{1}$, Shin-Kwang Khang ${ }^{2}$ \\ Departments of ${ }^{I}$ Neurological Surgery, ${ }^{2}$ Pathology, Asan Medical Center, College of Medicine, \\ University of Ulsan College of Medicine, Seoul, Korea
}

To report a case of spinal intramedullary cysticercosis in thoracic spine. A 47-year old man living in Korea referred to our hospital with both feet tingling sensation for about a year. Laboratory evaluations, including serologic tests were not helpful. Magnetic resonance imaging revealed a $1.7 \mathrm{~cm}$ intramedullary mass at T10-11 level, which believed to be a tumor instead, rather than a cysticercosis preoperatively. Successful operation was done with a histopathological result confirmed it as cysticercosis. Even though the prevalence of intramedullary spinal cysticercosis is extremely rare, and radiologic exams mimic other common tumors like ependymoma or astrocytoma, the disease should be considered as differential diagnosis.

Key Words: Cysticercosis • Intramedullary • Thoracic spine

\section{INTRODUCTION}

Neurocysticercosis, caused by Taenia solium, is the most common parasite infection in central nervous system. However, the prevalence of the disease is very rare; about $4 \%$ of the general population in endemic areas". Spinal cysticercosis represents about $1.2 \%$ to $5.8 \%$ of all cases of neurocysticercosis ${ }^{5,6}$. Spinal cysticercosis can be classified anatomically as extraspinal (vertebral) or intraspinal (epidural, subdural, arachnoid, or intramedullary), where intramedullary type is very rare. We report a case of intramedullary cysticercosis at T10-11 level treated surgically and suggest a treatment guideline.

\section{CASE REPORT}

A 47-year-old man, referred to our hospital with chief complaint of both feet tingling sensations for about a year. The symptom exacerbated after walking and the medication did not work. Subsequently, he developed progressive ambulation

\footnotetext{
- Received: April 12, 2014 - Revised: May 2, 2014

- Accepted: May 9, 2014

Corresponding Author: Seung Chul Rhim, MD, PhD

Department of Neurological Surgery, Asan Medical Center, University of Ulsan College of Medicine, 86 Asanbyeongwon-gil, Songpa-gu, Seoul 138-736, Korea Tel: +82-2-3010-3555, Fax: +82-2-476-6738

E-mail: aussie84@naver.com

@This is an Open Access article distributed under the terms of the Creative Commons Attribution Non-Commercial License (http://creativecommons.org/ licenses/by-nc/3.0/) which permits unrestricted non-commercial use, distribution, and reproduction in any medium, provided the original work is properly cited.
}

difficulty. He had no history of foreign travel in the past 10 years.

\section{Examination}

Neurologic examination showed motor power grade $4+/ 5$ in both lower limbs. Magnetic resonance imaging (MRI) study of the thoracic spine revealed heterogeneously enhancing intramedullary mass in T10-11 level, with peritumoral hemorrhage and central cystic change (Fig. 1). Differential diagnosis included cavernous malformation as multi-staged hemorrhage, but rather considered as ependymoma due to the solid enhancing portion in the mass.

The result of laboratory tests and measurements including electrolytes, C-reactive protein and erythrocyte sedimentation rate were normal. The peripheral leukocyte count was $7,400 / \mu \mathrm{L}$ with 3.5\% eosinophils. Serum enzyme-linked immunoelectric transfer blood assay for cysticercosis antibody revealed no abnormalities.

\section{Operations}

The patient underwent T9-11 laminoplastic laminotomy and the tumor was located intramedullary portion. Gross examination of the lesions was oval shaped, dark blue colored, not well encapsulated and showed moderate vascularity. The cyst contained clear fluid (Fig. 2).

\section{Pathologic Examination}

Histopathological examination of the cysticercosis cyst 

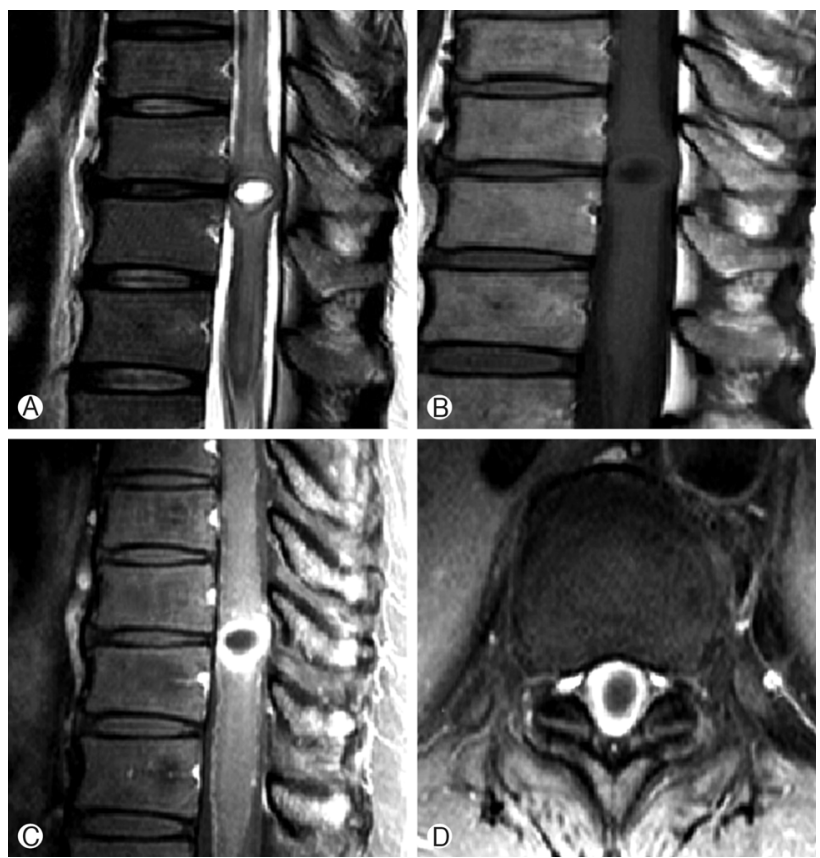

Fig. 1. Preoperative magnetic resonance images of the patient. (A) Sagittal T2-weighted (B) Sagittal T1weighted (C) Contrast-enhanced Sagittal T1 weighted. (D) Axial T2-weighted. About $1.7 \mathrm{~cm}$ intramedullary mass in T10-11 level. T2Wl shows heterogenous dark signal intensity and central cystic changes with strong enhance.

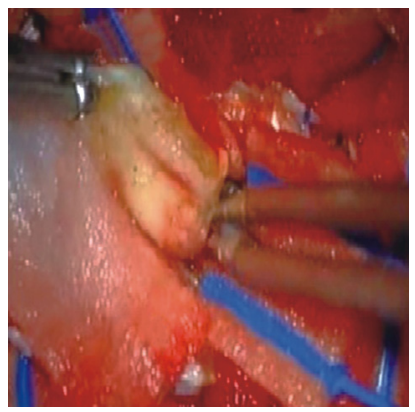

Fig. 2. Intraoperative photography. Laminiplstic laminotomy, T10, subtotal laminectomy T9 lower $\mathrm{T} 11$ upper and total removal of the mass.

with eosinophilic lining and clear fluid with chronic inflammatory cells (Fig. 3).

\section{Postoperative Course}

Postoperatively, patient's paresthesia showed no change but his muscle strength improved rapidly with rehabilitation program. Follow-up MRI showed no residual lesion. He was consulted with infection department and discharged from hospital with a prescription of Albendazole and dexamethasone. Computed tomography scan of the brain was taken and revealed a small calcified nodule in a parietal area, most likely cysticercosis (Fig. 4). 1 year follow up on OPD showed much

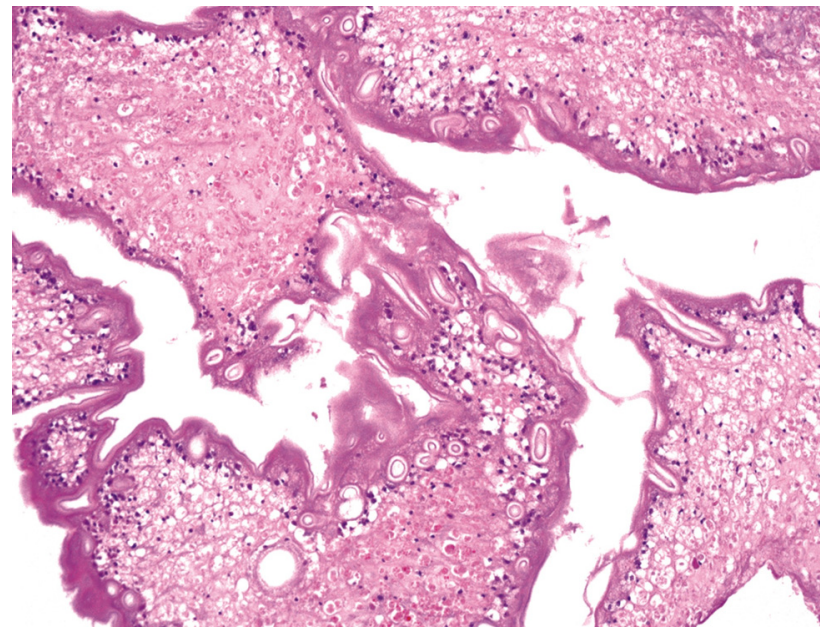

Fig. 3. Photomicrographs of histopathological specimen of the mass. Wall of the Worm shows convoluted Tegument and excretory canals. Hematoxylin and eosin, $\times 200$
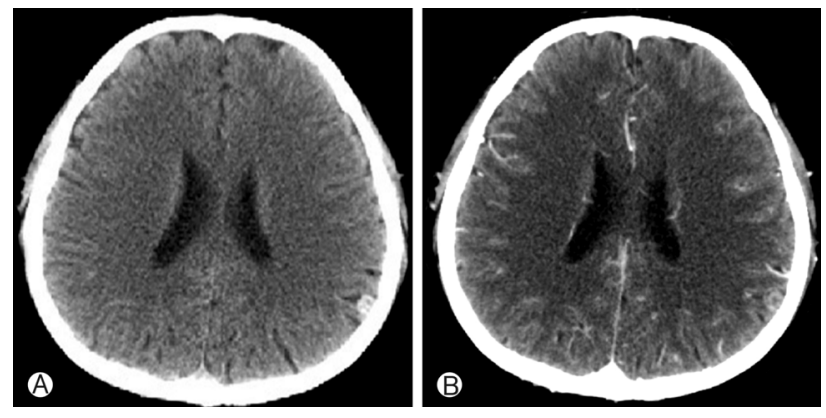

Fig. 4. Computed tomography scan of the brain revealed a small calcified nodule in a parietal area, most likely cysticercosis. (A) Non-enhance. (B) Contrast-enhanced.

improved general condition with no definite neurologic deficits.

\section{DISCUSSION}

Neurocysticercosis, caused by infection known as Taenia solium, is a common disease but only $1-5 \%$ of the cases are purely intraspinal ${ }^{9)}$. Although, it is a most common parasite infection in CNS, this disease entity is very rare in Republic of Korea, it is more endemic in countries like Latin America, Mexico and other southeast Asian countries ${ }^{2,3,6}$. The cause of this infection is known to be ingestion of cysticercal eggs in food contaminated by human or porcine feces ${ }^{2,9)}$.

It is difficult to clinically suspect spinal cysticercosis in the absence of previous history. Some findings like, high eosinophil count and calcification of soft tissues in the plain radiograms ${ }^{2)}$ can be helpful.

CSF results can be helpful, but not definite; moderate lym- 
phocytic pleocytosis, variable eosinophilic pleocytosis, elevated protein, and low or normal glucose level ${ }^{2}$. However lumbar puncture should be carefully managed as it may precipitate neurological deterioration in patients with history of spine treatment.

Diagnosis of cysticercosis is largely depending on histopathology results. The constellation of clinical history, exposure, serologic tests and radiologic studies can be helpful. The vesicular stage of neurocysticercosis appear as a well defined hypointense cyst with hyperintense scolex inside on the T1W ${ }^{14)}$. The thickened cyst capsule in the colloidal stage is hyperintense on T1WI and hypointense on T2-weighed image. During this stage, the cyst contents appear relatively hyperintense on T1WI which makes discrimination of the scolex not possible ${ }^{5,9,15)}$. The differential diagnosis of an intramedullary cystic lesion include arachnoid cyst, ependymal cyst, neurenteric cyst, sarcoidosis and neoplasms such as ependymoma ${ }^{10,14}$. Our case showed multi-stage hemorrhage, so ependymoma and cavernous malformation were considered first.

Our case was much unexpected because the patient lacked of neurocysticercosis history and was not from an endemic region. Having in mind more common pathology, such as intramedullary tumor or herniated disc, it was surprising that the disease was revealed as cysticercosis. A differential diagnosis of intramedullary spinal cysticercosis should be entertained in cases of unusual radiologic findings, which include focal cystic lesion with enhancement and cord edema extending over some segments, which may be hard to differentiat from other prevalent tumors like astrocytoma or ependymoma.

If the diagnosis is doubtful but patients neurologic deterioration worsens, surgery should be considered ${ }^{2)}$. Surgery was a good choice in our case in order to remove the mass and thereby decompressing the spinal cord and confirm the diagnosis. The patient showed improvement in motor power. The results of surgery are not definite. Some old reports show high mortality $(15 \%)$ and morbidity (85\%) with surgery ${ }^{15)}$. However, recent studies showed significantly improved surgical outcome; no death were reported and most of patients were likely to live without major neurologic deficits postoperatively ${ }^{1,2,9,11,15)}$.

Medical treatment instead of surgery is considered only for patients with no progressive neurologic deficits and highly suspected as intramedullary cysticercosis ${ }^{2,3,8)}$. Albendazole is treatment of choice as a regular treatment. Corticosteroids are often used together as it increases albendazole blood levels and may reduce the inflammatory reactions associated with the treatment ${ }^{7}$. Cerebral involvement concurrently with spinal cysticercosis is very high ${ }^{4,13)}$. Cerebral involvement may result in hydrocephalus and consequently increase ICP. Therefore early management is mandatory in most of cases ${ }^{12}$.

\section{CONCLUSIONS}

The prevalent disease should be considered first in most of cases. However like in our case, when radiologic exams were much more like ependymoma or cavernous malformation and patients' clinical symptoms failed to prove it as cysticercosis, the disease should be considered as differential diagnosis. If patient is stable, medical treatment could be considered, but as the diagnosis mostly requires histopathology results and patients neurologic deterioration occurs, surgery is the treatment of choice to facilitate the eradicate the lesion, decompress the spinal cord and confirm the diagnosis as thereby prove the better outcome.

\section{REFERENCES}

1. Agrawal R, Chauhan SP, Misra V, Singh PA, Gopal NN: Focal spinal intramedullary cysticercosis. Acta Biomed 79:39-41, 2008

2. Ahmad FU, Sharma BS: Treatment of intramedullary spinal cysticercosis: report of 2 cases and review of literature. Surg Neurol 67:74-77; discussion 77, 2007

3. Apuzzo ML, Dobkin WR, Zee CS, Chan JC, Giannotta SL, Weiss $\mathrm{MH}$ : Surgical considerations in treatment of intraventricular cysticercosis. An analysis of 45 cases. J Neurosurg 60:400-407, 1984

4. Barry M, Kaldjian LC: Neurocysticercosis. Semin Neurol 13:131143, 1993

5. Castillo M, Quencer RM, Post MJ: MR of intramedullary spinal cysticercosis. AJNR Am J Neuroradiol 9:393-395, 1988

6. Colli BO, Assirati Junior JA, Machado HR, dos Santos F, Takayanagui OM: Cysticercosis of the central nervous system. II. Spinal cysticercosis. Arq Neuropsiquiatr 52:187-199, 1994

7. Corral I, Quereda C, Moreno A, Lopez-Velez R, Martinez-SanMillan J, Guerrero A, et al: Intramedullary cysticercosis cured with drug treatment. A case report. Spine (Phila Pa 1976) 21: 2284-2287, 1996

8. Couldwell WT, Zee CS, Apuzzo ML: Definition of the role of contemporary surgical management in cisternal and parenchymatous cysticercosis cerebri. Neurosurgery 28:231-237, 1991

9. Gaur V, Gupta RK, Dev R, Kathuria MK, Husain M: MR imaging of intramedullary spinal cysticercosis: A report of two cases. Clin Radiol 55:311-314, 2000

10. Iwahashi H, Kawai S, Watabe Y, Chitoku S, Akita N, Fuji T, et al: Spinal intramedullary ependymal cyst: a case report. Surg Neurol 52:357-361, 1999

11. Kasliwal MK, Gupta DK, Suri V, Sharma BS, Garg A: Isolated spinal neurocysticercosis with clinical pleomorphism. Turk Neurosurg 18:294-297, 2008

12. Keane JR: Death from cysticercosis. Seven patients with unrecognized obstructive hydrocephalus. West J Med 140:787-789, 1984

13. Leite CC, Jinkins JR, Escobar BE, Magalhaes AC, Gomes GC, 
Dib G, et al: MR imaging of intramedullary and intraduralextramedullary spinal cysticercosis. AJR Am J Roentgenol 169: 1713-1717, 1997

14. Lmejjati M, Aniba K, Haddi M, Hakkou M, Ghannane H, Ait Ben Ali S: Spinal intramedullary arachnoid cyst in children.
Pediatr Neurosurg 44:243-246, 2008

15. Mathuriya SN, Khosla VK, Vasishta RK, Tewari MK, Pathak A, Prabhakar S: Intramedullary cysticercosis: MRI diagnosis. Neurol India 49:71-74, 2001 